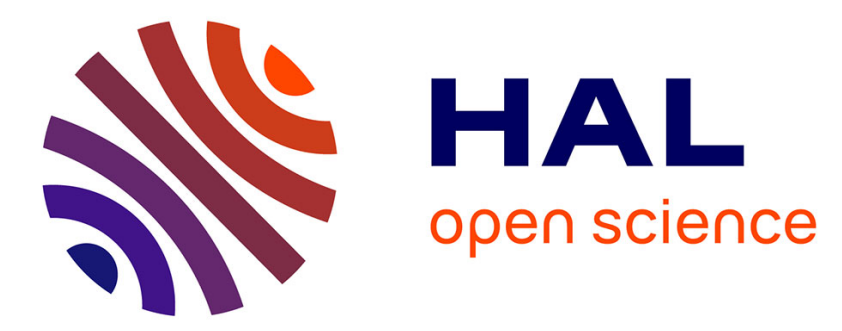

\title{
Performance Analysis of Extended RASK under Imperfect Channel Estimation and Antenna Correlation
}

Ali Mokh, Matthieu Crussière, Jean-Christophe Prévotet

\section{To cite this version:}

Ali Mokh, Matthieu Crussière, Jean-Christophe Prévotet. Performance Analysis of Extended RASK under Imperfect Channel Estimation and Antenna Correlation. IEEE Wireless Communications and Networking Conference, Apr 2018, Barcelona, Spain. 10.1109/wcnc.2018.8377261 • hal-01722204

\section{HAL Id: hal-01722204 \\ https://hal.science/hal-01722204}

Submitted on 3 Mar 2018

HAL is a multi-disciplinary open access archive for the deposit and dissemination of scientific research documents, whether they are published or not. The documents may come from teaching and research institutions in France or abroad, or from public or private research centers.
L'archive ouverte pluridisciplinaire HAL, est destinée au dépôt et à la diffusion de documents scientifiques de niveau recherche, publiés ou non, émanant des établissements d'enseignement et de recherche français ou étrangers, des laboratoires publics ou privés. 


\title{
Performance Analysis of Extended RASK under Imperfect Channel Estimation and Antenna Correlation
}

\author{
Ali Mokh, Matthieu Crussière, Maryline Hélard \\ Univ Rennes, INSA Rennes, IETR, CNRS, UMR 6164, F-35000 Rennes
}

\begin{abstract}
Spatial modulations (SM) use the index of the transmit (or the receive) antennas to allow for additional spectral efficiency in MIMO systems by transmitting spatial data on top of classical IQ modulations. Extended Receive Antenna Shift Keying (ERASK) exploits the SM concept at the receiver side and yields the highest overall spectral efficiency in terms of spatial bits compared to the conventional SM schemes. To perform efficiently, ERASK may use zero-forcing precoding to target spatial streams towards the selected receive antennas, therefore requiring the Channel State Information CSI at the transmitter. In this paper, we evaluate the theoretical performance degradation of the ERASK scheme under imperfect CSI. In addition, correlation between antennas at the transmitter and the receiver using the Kronecker correlation model is integrated in our analysis. Analytical approach for the Bit Error Rate performance is provided and validated through simulations.

Index Terms-ERASK, MIMO, Spatial Modulation, Space Shift Keying, Zero Forcing, Channel Estimation Error, channel correlation
\end{abstract}

\section{INTRODUCTION}

Since their introduction about 20 years ago, multiple-input and multiple-output (MIMO) wireless systems have been proved to allow for an impressive increase in system capacity in the presence of multipath fading environments [1].Indeed, MIMO technology represents today one of the major steps in the enhan cement of many wireless communication systems [2]. One sub-branch of MIMO schemes referred to as Spatial Modulation (SM) appeared in the early 2000s, with the idea of exploiting the index of the transmit (TA) or receive antennas (RA) to transmit information bits. In such techniques, transmit or receive antenna selection is used as a spatial mapping function to carry information bits over a MIMO channel in addition to common IQ symbols.

SM schemes have initially been designed to activate one single spatial stream at a time, thereby overcoming interchannel interference and considerably reducing the radiofrequency chain complexity [3]. One of the first proposed SM scheme known as space shift keying (SSK) [4][5], applies the SM concept at the transmitter side by simply selecting one TA out of $N_{t}$ to transmit $\log _{2} N_{t}$ spatial bits. The main idea behind such a concept is to recognize spatial bits from the various propagation signatures of the spatial streams associated to each TA activation [6]. With SSK hence, the receiver has to exhaustively learn the whole set of spatial signatures before being able to process the spatial demodulation. The SSK concept can also be applied at the receiver side thus leading to the so-called Receive-Spatial Modulation (RSM) [7] or Receive Antenna Shift Keying (RASK) [8] schemes. In such cases, one out of $N_{r}$ RA is targeted (instead of being activated) and the index of the targeted RA carries the additional spatial bits, thus yielding a spectral efficiency of $\log _{2} N_{r}$. RSM and RASK hence rely on particular preprocessing techniques able to focus the propagated waves to the selected RA, such as Time Reversal (TR), Zero-Forcing (ZF) or other beamforming schemes [8][9][10]. As such, RSM schemes assume that the MIMO channel response is known at the transmitter. From a receiver point of view however, spatial demodulation amounts to simply detecting the targeted antenna which is considered as a low-complexity processing compared to conventional MIMO detection schemes [3].

A generalization of the RSM principle, further referred to as GPSM (Generalised Pre-coding aided Spatial Modulation), is proposed in [11] where the TA array concentrates the signal energy towards a subset of RAs of fixed size $N_{a} \geq 2$. This allows the SM-MIMO system to reach an increased spectral efficiency compared to conventional SM schemes, but, opposed to them where a single RF chains is deployed, all the RAs have to be active (in parallel or through switches) with generalized RSM in order to enable the detection of the subset of targeted RAs. Ultimately extending the concept of RSM by enabling all possible combinations of different numbers of targeted antennas leads to the ERASK (Extended RASK) scheme as proposed in [12]-[14]. Eventually, ERASK yields the highest possible spectral efficiency (spatial bits) for a SM scheme, i.e. $N_{r}$, while being possibly applied with reasonable decoding complexity through a threshold detector at the receiver side.

As any RSM system, ERASK however relies on beamforming techniques meaning that channel state information (CSI) is needed at the transmitter side. In real cases, supplying the transmitter with accurate CSIT is a difficult task. The negative effects of channel estimation errors on the performance of SM at the transmitter when operating over flat Rayleigh fading channels is investigated in [15], and authors in [7] addressed the problem of imperfect or partial CSIT on the transmit precoding for RSM. In this paper, we evaluate the effect of imperfect CSIT on the ERASK scheme assuming ZF preprocessing and using a real amplitude threshold as proposed and justified in [12]. In addition, we integrate in our model the impact of antenna correlation which may also have strong im- 


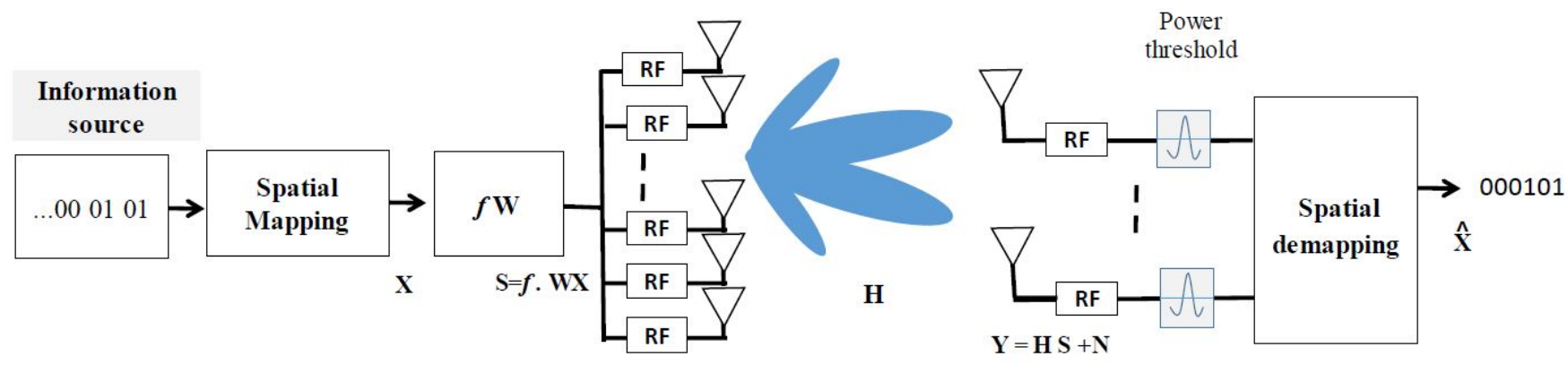

Figure 1. Block diagram of Extended-RASK

pact on the performance of SM systems. Theoretical Bit Error Rate (BER) is derived and validated through simulations, and various MIMO topologies with different number of antennas are compared giving many insights on the sensitivity of the ERASK scheme to imperfect CSIT and antenna correlation.

The rest of the paper is organized as follows. In Section II, the system model and the block diagram of the ERASK scheme are detailed. The models for imperfect CSIT and for transmit/receive spatial correlation are modeled in Section III. The theoretical computation of the BER is detailed in Section IV. Simulation results are provided in Section V, and a conclusion is drawn in Section VI.

\section{SYSTEM MODEL}

In this section, we set up the model for a communication system making use of the ERASK scheme. As any SM scheme, ERASK is based on a classical MIMO system topology. We denote by $N_{t}$ the number of TAs and $N_{r}$ the number of RAs. Assuming flat fading channels between the transmitter and the receiver, the input-ouput matrix form signal representation involving all the spatial links of the MIMO channel is commonly written as:

$$
\mathbf{Y}=\mathbf{H ~ S}+\mathbf{N}
$$

where $\mathbf{S} \in \mathbb{C}^{N_{t} \times 1}$ and $\mathbf{Y} \in \mathbb{C}^{N_{r} \times 1}$ are the transmit and receive symbol vectors and $\mathbf{H} \in \mathbb{C}^{N_{r} \times N_{t}}$ is the MIMO channel matrix with elements $H_{j, i}$ representing the complex channel coefficient between the $i$ th transmit antenna $T_{i}$, and the $j$ th receive antenna $R_{j}$. Finally, $\mathbf{N} \in \mathbb{C}^{N_{r} \times 1}$ is the vector of additive white Gaussian noise (AWGN) samples $\eta_{j}$ such that $\eta_{j} \sim \mathcal{C N}\left(0, \sigma_{n}^{2}\right)$.

On that basis, SM schemes define particular mechanisms to exploit the MIMO channel to map symbols onto spatial links. Fig. 1 presents the block diagram of the studied ERASK system. With ERASK, a group of $m=N_{r}$ bits is associated to a spatial symbol $\mathbf{X} \in \mathbb{N}^{N_{r} \times 1}$ which is written as:

$$
\mathbf{X}=\left[\begin{array}{llll}
x_{1} & x_{2} & \ldots & x_{N_{r}}
\end{array}\right]^{T} \text { where } x_{j} \in\{0, A\},
$$

each $x_{j}$ entry determining the set of targeted RAs such that:

$$
x_{j}= \begin{cases}0 & \text { if } R_{j} \text { is not targeted } \\ A & \text { if } R_{j} \text { is targeted. }\end{cases}
$$

Contrary to other RSM schemes, ERASK considers any combinations of targeted antennas, so that each antenna have the same probability to be targeted or not, i.e. $\frac{1}{2}$. The target mechanism is then obtained through the pre-processing block which transforms the vector of spatial symbols $\mathbf{X}$ into the vector of transmitted signals $\mathbf{S} \in \mathbb{C}^{N_{t} \times 1}$ as,

$$
\mathbf{S}=f \mathbf{W} \mathbf{X}
$$

where $\mathbf{W} \in \mathbb{C}^{N_{t} \times N_{r}}$ is the pre-processing matrix, of entries $w_{i, j}, i \in\left[\begin{array}{ll}1 & N_{t}\end{array}\right]$ and $j \in\left[\begin{array}{ll}1 & N_{r}\end{array}\right]$ and $f$ is a normalization factor defined as,

$$
f=\frac{1}{\sqrt{\sigma_{x}^{2} \operatorname{Tr}\left(\mathbf{W} \mathbf{W}^{H}\right)}}
$$

where $\operatorname{Tr}($.$) holds for the trace of matrix and \sigma_{x}^{2}=\mathbb{E}_{x}\left[x_{j} x_{j}^{*}\right]$ is independent on $j$ since $\mathbf{X}$ has i.i.d. entries. As each entry of $\mathbf{X}$ is of amplitude $A$ with a probability $\frac{1}{2}$, we can further state that $\sigma_{x}^{2}=\frac{A^{2}}{2}$.

As argued in [12], ZF beamforming is the best preprocessing strategy for ERASK. Indeed, supposing that the number of antennas satisfies the constraint $N_{r} \leq N_{t}$, it can annihilate all interference on the received signal and then leads to the best antenna detection performance. Assuming perfect CSIT, $\mathbf{W}=\mathbf{H}^{\dagger}\left(\mathbf{H H}^{\dagger}\right)^{-1}$ so that the received signal vector is given by,

$$
\mathbf{Y}=f \mathbf{X}+\mathbf{N}
$$

and the received signal $y_{j}$ at antenna $R_{j}$ then writes,

$$
y_{j}=f \times x_{j}+\eta_{j} .
$$

Hence, under perfect CSIT assumption, no interference is added to the received signal at each RA, and a simple amplitude threshold detector can be used to recover the spatial symbols by detecting whether each RA is targeted by the transmit array or not. One may use a coherent detector as proposed in [12] leading to a demodulation process carried out through an independent and parallel signal analysis per antenna. Considering that the target signal $x_{j}=A$ is of known phase $\phi_{A}$ at the receiver, the receiver can compensate the phase 
of the received signal and compare the real part obtained to a predefined amplitude threshold $\nu$ as,

$$
\hat{x}_{j}= \begin{cases}0, & \text { if } \Re\left\{y_{j} \times e^{-\phi_{A}}\right\} \leq \nu, \\ A, & \text { if } \Re\left\{y_{j} \times e^{-\phi_{A}}\right\} \geq \nu .\end{cases}
$$

where the threshold is [12]:

$$
\nu=\frac{f \cdot A}{2} .
$$

From such antenna detection process, a simple look-up table based spatial demapping is finally employed to decode the binary information.

\section{IMPERFECT CSIT AND CHANNEL CORRELATION}

In the sequel, we first introduce the channel state information error model and its effect on the received signal. Then, antenna correlation at the transmitter and the receiver is incorporated in our model. Finally, the resulting received signal expression in given.

\section{A. Imperfect CSIT}

A perfect channel knowledge at any side of the transmission system is impossible in practical situations. In this paper, the transmitter is assumed to be capable of accurately tracking the long term average Channel State Information (CSI). This can be for instance plausible in time-division duplexing (TDD) mode, where the reciprocity of the channel can apply. However, we consider in the sequel that an instantaneous CSI error is added to the long term channel estimation, such that we have,

$$
\mathbf{H}=\overline{\mathbf{H}}+\tilde{\mathbf{H}}
$$

where $\overline{\mathbf{H}}$ represents the average long term CSI that is accurately estimated at the transmitter, and $\tilde{\mathbf{H}}$ denotes the instantaneous CSI deviation matrix where all entries obey the complex Gaussian distribution $\tilde{h}_{j, i} \sim \mathcal{C N}\left(0, \sigma_{H}^{2}\right)$. Since $\overline{\mathbf{H}}$ is estimated at the transmitter, then the pseudo-inverse of $\overline{\mathbf{H}}$ is used as a pre-filter as,

$$
\mathbf{W}=\overline{\mathbf{H}}^{\dagger}\left(\overline{\mathbf{H}} \overline{\mathbf{H}}^{\dagger}\right)^{-1} .
$$

\section{B. Transmit and Receive Spatial Correlation}

Performance of SM schemes are often evaluated over a independent Rayleigh fading per antenna link. In this work the correlation between TAs and between RAs is taken into account to evaluate the robustness of the system ERASK. Employing the Kronecker correlation model [16] for that purpose, the MIMO channel can be rewritten as:

$$
\mathbf{H}=\mathbf{R}_{r}^{1 / 2} \mathbf{H}_{I}\left(\mathbf{R}_{t}^{1 / 2}\right)^{T}
$$

where $\mathbf{H}_{I} \sim \mathcal{C N}(\mathbf{0}, \mathbf{I})$ is the channel matrix with independent entries, and $\mathbf{R}_{r}$ and $\mathbf{R}_{t}$ represent the receive and transmit spatial correlation matrices at the receive and transmit arrays, respectively. The correlation matrices are generated using the exponential model, such that $\mathbf{R}_{r}(i, j)=\rho_{r}^{|i-j|}$ and $\mathbf{R}_{t}(i, j)=$ $\rho_{t}^{|i-j|}$, where $0 \leq \rho_{r}, \rho_{t} \leq 1$. Then combining the correlation model with the imperfect channel estimation model, we may write the MIMO channel as:

$$
\mathbf{H}=\underbrace{\mathbf{R}_{R}^{1 / 2} \overline{\mathbf{H}}_{I}\left(\mathbf{R}_{T}^{1 / 2}\right)^{T}}_{\tilde{\mathbf{H}}}+\underbrace{\mathbf{R}_{R}^{1 / 2} \tilde{\mathbf{H}}_{I}\left(\mathbf{R}_{T}^{1 / 2}\right)^{T}}_{\tilde{\mathbf{H}}}
$$

where matrix $\overline{\mathbf{H}}$ represents the full known part of the channel and matrix $\tilde{\mathbf{H}}$ represents the unknown part of the channel, both integrating correlation effects.

\section{Received Signal and Detection Algorithm}

From the above model, in which the estimation of the channel includes errors, the equation of the received signal (5) becomes :

$$
\mathbf{Y}=f \mathbf{X}+f \tilde{\mathbf{H}} \mathbf{W} \mathbf{X}+\mathbf{N}
$$

which can be re-expressed at each received antenna as,

$$
y_{j}=f \times x_{j}+f \times \sum_{k=1}^{N_{r}} \sum_{i=1}^{N_{t}} \tilde{h}_{j, i} w_{i, k} x_{k}+\eta_{j} .
$$

As expected from the erroneous channel knowledge, ZF beamforming fails in properly isolating the entries of the spatial symbol vector which gives rise to a residual interference term in the latter two expressions.

\section{PERformance AnAlysis}

Based on the obtained receive signal in Eq. (14), we provide in this section the analytical derivation for the theoretical BER performance of the ERASK system under imperfect channel estimation. The generic expression of the BER $\mathcal{P}_{e}$ can be written as,

$$
\mathcal{P}_{e}=\frac{1}{m} \cdot \mathbb{E}\left\{\sum_{k}^{N_{r}} \sum_{j \neq k}^{N_{r}} \mathcal{P}\left(\mathbf{X}_{k} \rightarrow \mathbf{X}_{j}\right) \cdot d\left(\mathbf{X}_{k}, \mathbf{X}_{j}\right)\right\} .
$$

where $d\left(\mathbf{X}_{k}, \mathbf{X}_{j}\right)$ is the Hamming distance between two spatial symbols $\mathbf{X}_{k}$ and $\mathbf{X}_{j}$, and $\mathcal{P}\left(\mathbf{X}_{k} \rightarrow \mathbf{X}_{j}\right)$ is the probability to transmit $\mathbf{X}_{k}$ and detect $\mathbf{X}_{j}$. All spatial signatures are possible and equally likely in ERASK scheme, so that for each RA, the probability of being targeted or not is independent on the fact that any other RA is also targeted. Consequently, evaluating the global BER of the ERASK system amounts to get the BER on each RA antenna. Let us define $\mathcal{P}\left(y_{j 1}\right)$ (resp. $\mathcal{P}\left(y_{j 0}\right)$ ) as the probability that one particular antenna $R_{j}$ is targeted (resp. not targeted), and $\mathcal{P}\left(y_{j 0} \rightarrow y_{j 1}\right)$ (resp. $\mathcal{P}\left(y_{j 1} \rightarrow y_{j 0}\right)$ the conditional probability that one particular antenna $R_{j}$ is detected as being targeted (not targeted) knowing that it was not (resp. it was). We then have,

$$
\mathcal{P}_{e}=\mathcal{P}\left(y_{j 0}\right) \cdot \mathcal{P}\left(y_{j 0} \rightarrow y_{j 1}\right)+\mathcal{P}\left(y_{j 1}\right) \cdot \mathcal{P}\left(y_{j 1} \rightarrow y_{j 0}\right)
$$

Since $x_{k} \in\{0, A\}$ with a probability of $\frac{1}{2}$, we have $\mathcal{P}\left(y_{j 0}\right)=$ $\mathcal{P}\left(y_{j 1}\right)=\frac{1}{2}$. In addition, following the threshold criteria introduced in Eq. (8), We can then simply restate the BER expression as,

$$
\mathcal{P}_{e}=\frac{1}{2} \cdot \mathcal{P}\left(y_{j 0}>\frac{f \cdot A}{2}\right)+\frac{1}{2} \cdot \mathcal{P}\left(y_{j 1}<\frac{f \cdot A}{2}\right) .
$$


The probabilities of false detection in Eq. (17) have to be analyzed from the expression of the received signal at antenna $R_{j}$ given in Eq. (6). It is important to keep in mind that the interference term embedded in Eq. (6) depends on the other targeted antennas. It can be statistically represented as,

$$
\begin{gathered}
f \times \sum_{k=1}^{N_{r}} \sum_{i=1}^{N_{t}} \tilde{h}_{j, i} w_{i, k} x_{k} \sim \mathcal{C N}\left(0, \sigma_{l}^{2}\right) . \\
\text { where } \sigma_{l}^{2}=\sum_{k=1}^{N_{r}} x_{k}^{2} \sum_{i=1}^{N_{t}} f^{2}\left\|w_{i, k}\right\|^{2} \sigma_{H}^{2} .
\end{gathered}
$$

Now expressing the normalization factor $f$ as,

$$
f^{2}=2 /\left(A^{2} \times \sum_{i=1}^{N_{t}} \sum_{j=1}^{N_{r}}\left\|w_{i, j}\right\|^{2}\right)
$$

the expression of the interference variance in (19) becomes:

$$
\sigma_{l}^{2}=\sum_{k=1}^{N_{r}} x_{k}^{2} \frac{2}{A^{2} \times N_{r}} \sigma_{H}^{2}
$$

in average over all possible spatial symbols. We can then decompose all possible spatial signatures to determine the distribution of the interference and the additive white noise depending on the number $N_{a}$ of targeted antennas as,

$$
\sigma_{l}^{2}=2 \sigma_{H}^{2} \times N_{a} / N_{r},
$$

where $0 \leq N_{a} \leq N_{r}$. Then, defining $\mathcal{P}\left(N_{a}\right)$ as the probability of error detection if $N_{a}$ antennas are targeted, we have from Eq. (17):

$$
\begin{aligned}
\mathcal{P}\left(N_{a}\right) & =\frac{1}{2} \cdot \mathcal{P}\left(\Re\left\{f \cdot A+\mathcal{C N}\left(0, \sigma_{l}^{2}+\sigma_{n}^{2}\right)\right\}<\frac{f \cdot A}{2}\right) \\
& +\frac{1}{2} \cdot \mathcal{P}\left(\Re\left\{\mathcal{C N}\left(0, \sigma_{l}^{2}+\sigma_{n}^{2}\right)\right\}>\frac{f \cdot A}{2}\right) \\
& =Q\left(f . A / 2 \sqrt{\sigma_{n}^{2}+\sigma_{l}^{2}}\right)
\end{aligned}
$$

where $Q($.$) denotes the Gaussian Q-function. As a result, the$ general equation of the BER taking every subset of targeted antennas is given as:

$$
\mathcal{P}_{e}=\frac{1}{2^{N_{r}}} \sum_{N_{a}=0}^{N_{r}} C_{N_{r}}^{N_{a}} Q\left(f . A / 2 \sqrt{\sigma_{n}^{2}+2 \sigma_{H}^{2} \times N_{a} / N_{r}}\right)
$$

where $C_{N_{r}}^{N_{a}}$ is the binomial coefficient giving the number of subsets of $N_{a}$ elements of a set of $N_{r}$ elements. Finally introducing the ratio between the average transmit power level to the interference and noise level as:

$$
\mathrm{SNR}=\frac{\bar{P}_{t}}{\sigma_{n}^{2}+\sigma_{l}^{2}}=\frac{1}{\sigma_{n}^{2}+\sigma_{l}^{2}},
$$

the equation of the BER performance given in Eq. (24) becomes:

$$
\mathcal{P}_{e}=\frac{1}{2^{N_{r}}} \sum_{N_{a}=0}^{N_{r}} C_{N_{r}}^{N_{a}} Q\left(\frac{f \cdot A}{2} \sqrt{S N R}\right)
$$

Such a result indicates that the system performance is driven by $f$, which directly depends on the MIMO channel characteristics, the number of RAs and the deviation of the channel estimation error (embedded in the SNR). Hence, it can already been anticipated that the BER will degrade as :

- $f$ decreases. This will occur as the antenna correlation increases, since $f$ becomes weak when the channel matrix is bad conditioned.

- $N_{r}$ increases. This translates the fact that a higher amount of antenna interference has to be canceled with large receive antenna arrays.

- $\sigma_{l}^{2}$ increases. This is a direct consequence of the channel estimation impairments.

\section{Simulation RESUlts}

The performance of the ERASK system based on a parallel threshold detection and under imperfect CSIT is evaluated through the measurement of the BER versus SNR. More precisely, in the following curves the variance of the CSIT deviation is set to a constant value and the level of the Gaussian noise at the receiver changes to illustrate the BER in terms of the SNR. It is assumed that $\overline{\mathbf{H}}$ is a MIMO flat fading channel matrix where $\bar{h}_{j, i}$ are complex coefficients following i.i.d. Rayleigh distribution, and the entries of the matrix of instantaneous CSI deviation are complex coefficients following the complex Gaussian distribution with a variance $\sigma_{H}^{2}$. The power for each sub-channel is normalized as,

$$
E\left[\left\|\bar{h}_{j, i}\right\|^{2}\right]+\sigma_{H}^{2}=1,
$$

and the correlated model described in Eq. (12) is used for various values of $\rho$. Simulations are carried out by implementing a sufficient number of iterations for different channel realizations.

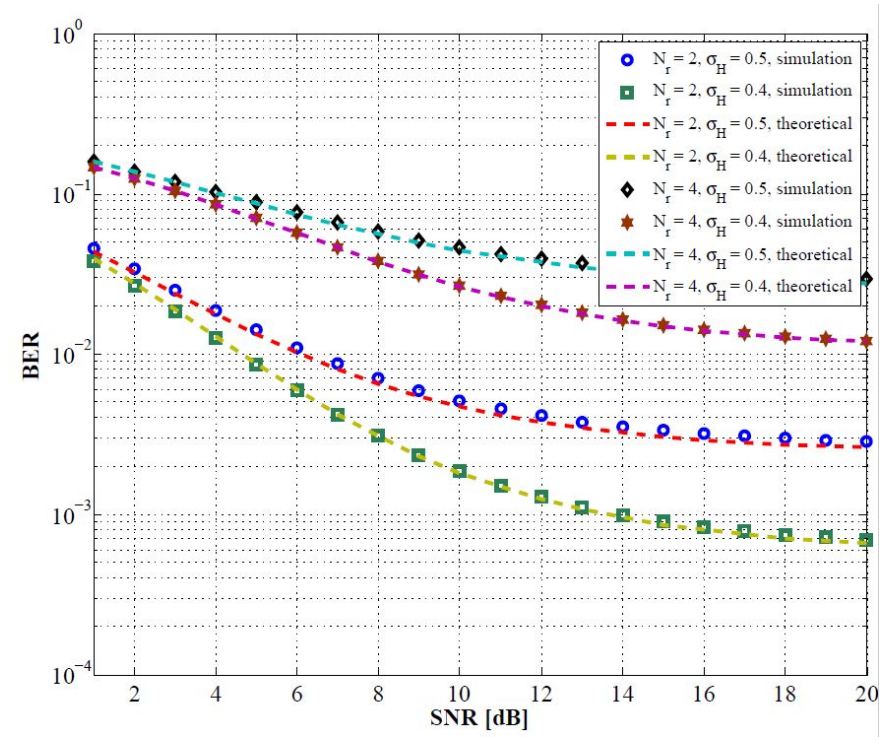

Figure 2. Theoretical and simulation comparison of BER Vs SNR performance of ERASK over uncorrelated Rayleigh fading channels. $N_{t}=8, N_{r}=2$ and $4, \sigma_{H}=0$ and 0.2 


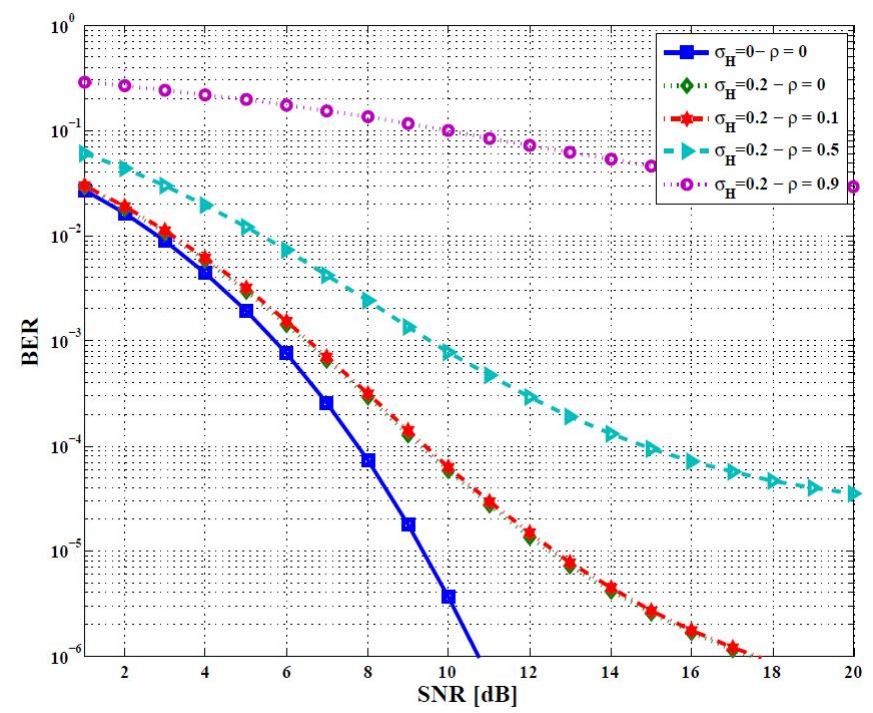

Figure 3. BER Vs SNR performance of ERASK with $N_{t}=8$ and $N_{r}=2$ over Rayleigh fading channels for perfect channel estimation and no correlation, and for $\sigma_{H}=0.2$ with correlation factor $\rho=0,0.1,0.5$ and 0.9 .

In Fig. 2, we first compare the simulated (markers) and theoretical (dashed lines) BER values for a ERASK system with $N_{t}=8$, and $N_{r}=2$ and 4 . For each $N_{r}$, results are given for two different channel estimation accuracies, namely $\sigma_{H}=$ 0.4 and 0.5. No antenna correlation is used in this figure. As evident from the obtained curves, theoretical results perfectly match simulation results for any configuration. Then, as expected from the analysis of Eq. (24), it is observed that increasing the order of the spatial modulation (i.e. increasing $N_{r}$ ), or degrading the CSIT quality leads to strong performance losses. In both cases indeed, the sum of added interference increases which translates into the form of error floors at high SNR.

The effect of the correlation at the transmitter and the receiver of the system, combined with the impact of channel estimation errors, is shown in Fig. 3. The considered ERASK system is set with $N_{t}=8$ and $N_{r}=2$. Two configurations are compared. The first one, used as a reference, assumes perfect CSIT and no correlation. The second one, considers a deviation of the estimation error of $\sigma_{H}=0.2$ and different correlation factor levels $(\rho=0,0.1,0.5$ and 0.9$)$. The ERASK performance obviously degrades when the correlation factor increases (lower $f$ ), and when channel estimation error is added (higher $\sigma_{l}^{2}$ ). Nevertheless, the degradation remains still acceptable for $\rho \leq 0.5$ with a performance degradation of less than $4 \mathrm{~dB}$ at a $10^{-3}$ BER compared to the perfect conditions, and less than $3 \mathrm{~dB}$ when only the channel estimation error is included $\left(\sigma_{H}=0.2, \rho=0\right)$. Interestingly, the curves obtained without correlation and with $\rho=0.1$ are almost undistinguished, which indicates that under a given quality of the channel estimation, the antenna correlation has no impact on the performance.

In Fig. 4, various ERASK system settings (i.e. various $N_{t} \times N_{r}$ ) are compared with and without channel estimation

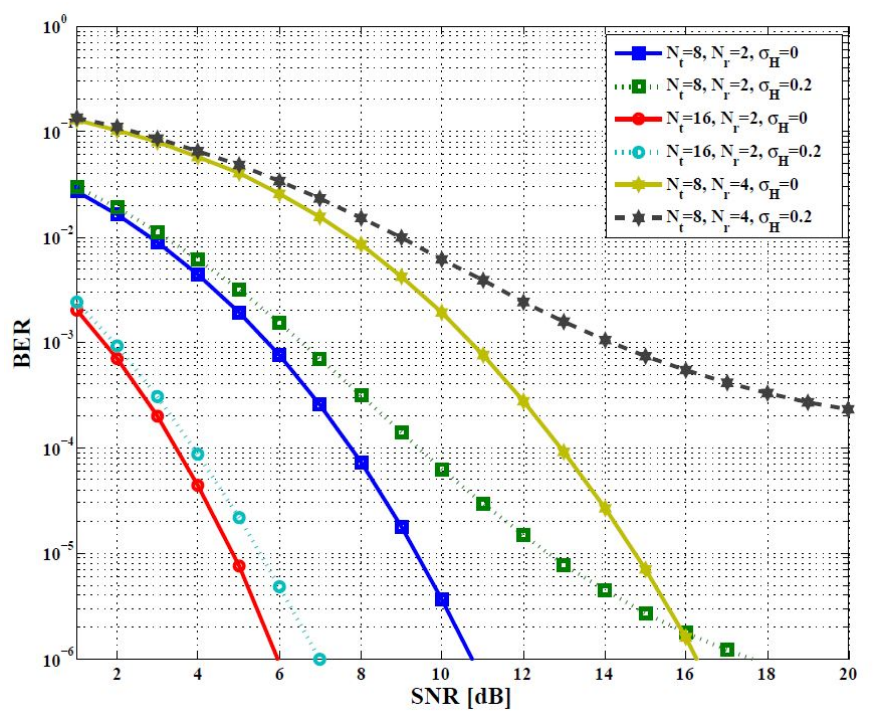

Figure 4. BER Vs SNR performance of ERASK over Rayleigh fading channels with antenna correlation factor $\rho=0.1 . N_{t}=8$ and $16, N_{r}=2$ and $4, \sigma_{H}=0$ and 0.2 .

inaccuracies, and fixing the correlation factor to $\rho=0.1$. First comparing the curves with $N_{t}=8$, it is verified that the larger the RA array, the stronger the degradation of the performance. Indeed, as the number of RAs increases, the amount of interantenna interference becomes higher at the receiver side and the defect of interference cancellation of the ZF scheme due the channel estimation errors becomes more significant. This fact corroborates the analysis made on Eq. (26). Reversely, analyzing the curves with $N_{r}=2$ but changing $N_{t}$ leads to the conclusion that the ZF beamforming is less sensitive to poor CSIT when a larger number of TAs is used. This indicates that using large transmit arrays, and thus exploiting higher spatial focusing gains, is favorable to channel estimation inaccuracies.

\section{CONCLUSION}

In this paper, we have evaluated the performance of the ERASK spatial modulation scheme based on ZF preprocessing under the impact of imperfect CSIT. We have followed an analytical approach to derive a closed-form expression of the BER of the ERASK scheme by integrating channel estimation errors in the system model. Also, the effect of the correlation between transmit antennas and receive antennas has been taken into account. Our theoretical results have been validated through simulations and have allowed for many insights in the analysis of the ERASK BER performance. Namely, it has be shown that the higher number of receive antennas, the stronger the degradation of the performance. However, the exposed results have also highlighted how more robust the ERASK scheme is against channel estimation impairments and antenna correlation when the number of transmit antennas increases. From our study, we can then conclude that the ERASK scheme can adapt many situations dealing with imperfect channel knowledge and antenna correlation as soon as some 
compensation is brought from the use of a sufficient number of transmit antennas.

\section{ACKNOWLEDGMENT}

The authors would like to thank the SPATIAL MODULATION project funded by the French National Research Agency (ANR).

\section{REFERENCES}

[1] J. H. Winters, J. Salz, and R. D. Gitlin, 'The impact of antenna diversity on the capacity of wireless communication systems', IEEE transactions on Communications, vol. 42, no. 234, pp. 1740-1751, 1994.

[2] G. Tsoulos, Mimo system technology for wireless communications. CRC press, 2006.

[3] M. Di Renzo, H. Haas, A. Ghrayeb, S. Sugiura, and L. Hanzo, 'Spatial modulation for generalized mimo: Challenges, opportunities, and implementation', Proceedings of the IEEE, vol. 102, no. 1, pp. 56-103, 2014.

[4] Y. A. Chau and S.-H. Yu, 'Space modulation on wireless fading channels', in Vehicular Technology Conference, 2001. VTC 2001 Fall. IEEE VTS 54th, IEEE, vol. 3, 2001, pp. 1668-1671.

[5] R. Y. Mesleh, H. Haas, S. Sinanovic, C. W. Ahn, and S. Yun, 'Spatial modulation', IEEE Transactions on Vehicular Technology, vol. 57, no. 4, pp. 2228-2241, 2008.

[6] J. Jeganathan, A. Ghrayeb, L. Szczecinski, and A. Ceron, 'Space shift keying modulation for mimo channels', IEEE Transactions on Wireless Communications, vol. 8, no. 7, pp. 3692-3703, 2009.

[7] A. Stavridis, S. Sinanovic, M. Di Renzo, and H. Haas, 'Transmit precoding for receive spatial modulation using imperfect channel knowledge', in Vehicular Technology Conference (VTC Spring), 2012 IEEE 75th, IEEE, 2012, pp. 1-5.
[8] D.-T. Phan-Huy and M. Hélard, 'Receive antenna shift keying for time reversal wireless communications', in 2012 IEEE International Conference on Communications (ICC), IEEE, 2012, pp. 4852-4856.

[9] L.-L. Yang, 'Transmitter preprocessing aided spatial modulation for multiple-input multiple-output systems', in Vehicular Technology Conference (VTC Spring), 2011 IEEE 73rd, IEEE, 2011, pp. 1-5.

[10] A. Mokh, Y. Kokar, M. Hélard, and M. Crussière, 'Time reversal receive antenna shift keying on mimo los channel', in 2017 International Conference on Sensors, Networks, Smart and Emerging Technologies (SENSET), IEEE, 2017.

[11] R. Zhang, L.-L. Yang, and L. Hanzo, 'Generalised precoding aided spatial modulation', IEEE Transactions on Wireless Communications, vol. 12, no. 11, pp. 5434 5443, 2013.

[12] A. Mokh, M. Hélard, and M. Crussière, 'Extended receive antenna shift keying', in 2017 IEEE International Conference on Telecommunication (ICT), IEEE, 2017.

[13] _ ' 'Space shift keying modulations for low complexity internet-of-things devices', in 2017 Global Communications Conference (GLOBECOM), IEEE, 2017.

[14] A. Mokh, M. Crussière, and M. Hélard, 'Performance analysis of the maximum ratio transmission preprocessing for extended receive antenna shift keying', in 2017 International Symposium on Wireless Personal Multimedia Communications (WPMC), IEEE, 2017.

[15] S. Sugiura and L. Hanzo, 'Effects of channel estimation on spatial modulation', IEEE Signal Processing Letters, vol. 19, no. 12, pp. 805-808, 2012.

[16] A. Forenza, D. J. Love, and R. W. Heath, 'A low complexity algorithm to simulate the spatial covariance matrix for clustered mimo channel models', in Vehicular Technology Conference, 2004. VTC 2004-Spring. 2004 IEEE 59th, IEEE, vol. 2, 2004, pp. 889-893. 